\title{
Influence of hypertension on serum concentration of type IV collagen antigens in streptozotocin-diabetic and non-diabetic rats
}

\author{
C. Hasslacher ${ }^{1}$, D. Brocks ${ }^{3}$, J. Mann'1, G. Mall ${ }^{2}$ and R. Waldherr ${ }^{2}$ \\ Departments of ${ }^{1}$ Internal Medicine and ${ }^{2}$ Pathology, University of Heidelberg, Heidelberg, and ${ }^{3}$ Hoechst AG, Frankfurt am Main, FRG
}

\begin{abstract}
Summary. The serum concentration of $7 \mathrm{~S}$ collagen was measured radioimmunologically as a marker of basement membrane type IV collagen synthesis in diabetic and nondiabetic rats with Goldblatt hypertension. In non-diabetic rats the $7 \mathrm{~S}$ collagen level was significantly raised after induction of hypertension $(51 \% ; p<0.001)$, and showed a positive correlation with relative heart weight as an integral parameter of hypertension $(r=0.63 ; p<0.01)$. In diabetic rats, which displayed a $7 \mathrm{~S}$ collagen concentration roughly 2.5 times as high as the metabolically normal animals, the $7 \mathrm{~S}$ collagen level was
\end{abstract}

$27 \%$ higher in the hypertensive animals $(p<0.01)$. There was no correlation with blood pressure or heart weight, but only a positive correlation with blood glucose $(r=0.51 ; p<0.05)$. The results indicate that haemodynamic alterations may alter basement membrane collagen metabolism. However, type IV collagen metabolism in diabetes is influenced to a greater extent by metabolic than by haemodynamic factors.

Key words: Diabetes, streptozotocin, hypertension, type IV collagen, $7 \mathrm{~S}$ collagen, basement membrane metabolism.
Recent experimental and clinical studies have underscored the role of haemodynamic factors in the development of diabetic microangiopathy [1-3]. The hyperperfusion of the capillaries and the raised intracapillary pressure which have been observed in kidney and other organs in diabetes $[4,5]$ may cause an increase of plasma protein extravasation and structural damage $[6$, 7]. However, a more marked expansion of glomerular mesangium in kidneys of hypertensive diabetic rats and a thickening of the capillary basement membrane in non-diabetic animals under conditions of increased intracapillary pressure has been described irrespective of the methods or conditions used for inducing hypertension [7-9]. This finding suggests that haemodynamic factors may further affect the metabolism of the capillary basement membrane. In the present paper we investigated the effect of hypertension in diabetic and non-diabetic rats on serum concentration of type IV collagen, which is a characteristic structural constituent of capillary basement membrane and of glomerular mesangium. The serum component resembles a fragment of type IV collagen, $7 \mathrm{~S}$ collagen, which is released into the circulation from existing basement membranes or newly formed intact type IV collagen which has not been deposited in tissue $[10,11]$. Thus, serum concentration of type IV collagen determined by a radioimmunoassay specific for the $\mathrm{N}$-terminal crosslinking domain (7S collagen) reflects the formation and turnover of basement membrane collagen of the body [10-12].

\section{Materials and methods}

Male Wistar rats (aged 5-6 weeks, $130-150 \mathrm{~g}$, Ivanovas, Kisslegg, FRG) were used throughout the study. All animals received chow pellets and tap water ad libitum. After induction of hypertension, a $2 \%$ sodium chloride solution was offered additionally to prevent the development of malignant hypertension [13].

\section{Induction of hypertension and diabetes}

Goldblatt-type hypertension was induced by placement of a silver clip (internal diameter $0.2-0.22 \mathrm{~mm}$ ) around the left renal artery. Systolic blood pressure was measured by a tail-plethysmographic method [14] 3, 4, 6 and 8 weeks later under slight ether anaesthesia. At each time, at least two blood pressure recordings were made to ensure reproducibility. Rats were described as "hypertensive" if blood pressure values were greater than $140 \mathrm{mmHg}$ at all times of measurement. The blood pressure of sham-operated rats was less than $130 \mathrm{mmHg}$ at all times.

Four weeks after renal clipping, one half of the hypertensive rats matched according to blood pressure received streptozotocin intraperitoneally ( $75 \mathrm{mg} / \mathrm{kg}$ body weight) for induction of diabetes. One half of the sham-operated normotensive rats were treated in the same way. Mean body weight of normotensive and hypertensive rats at the time of streptozotocin injection was $275 \pm 18$ and $263 \pm 20$, re- 
Table 1. Blood pressure, body weight, kidney weight, heart weight, blood glucose, and serum creatinine in the four experimental groups

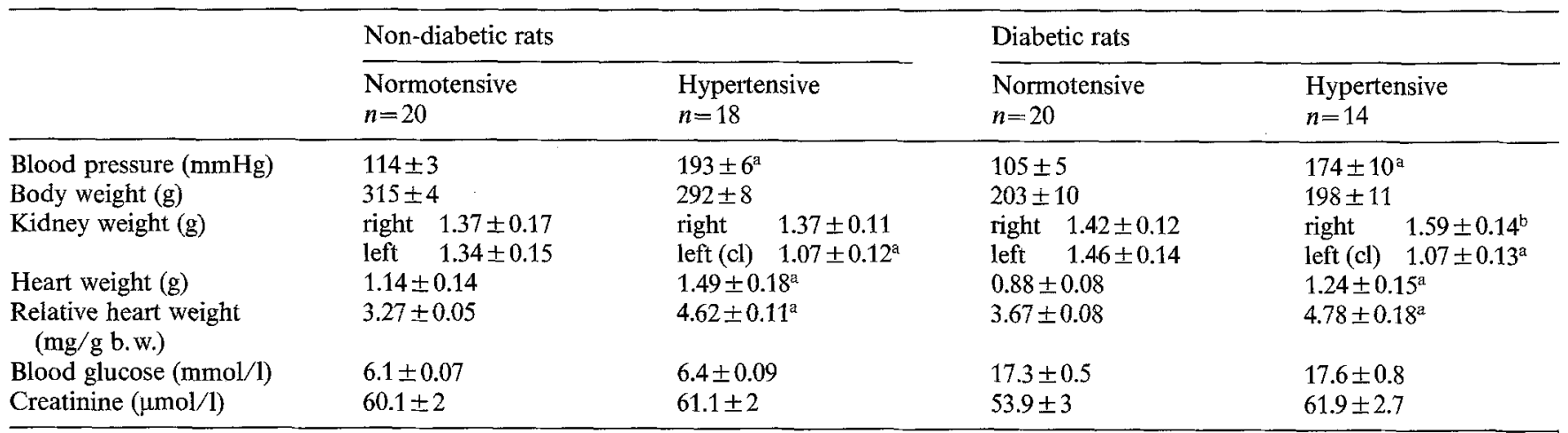

${ }^{\mathrm{a}} p<0.001$ compared to normotensive rats; ${ }^{\mathrm{b}} p<0.05$ compared to normotensive rats. $\mathrm{b} . \mathrm{w} .=$ body weight; $\mathrm{cl}=$ clipped. Results given as mean \pm SEM
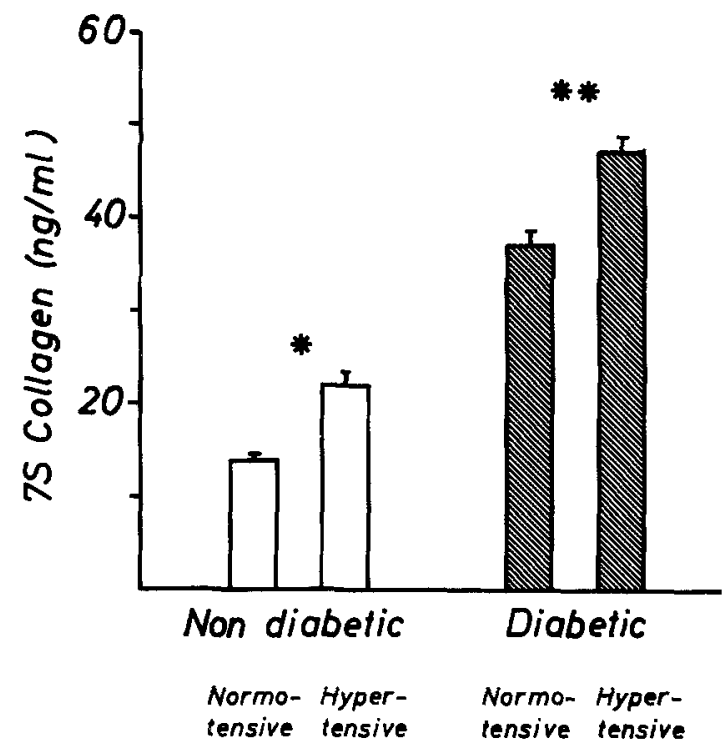

Fig. 1. Mean serum concentration of $7 \mathrm{~S}$ collagen $( \pm S E M)$ in nondiabetic and diabetic rats with and without hypertension. ${ }^{*} p<0.001$; ** $p<0.01$

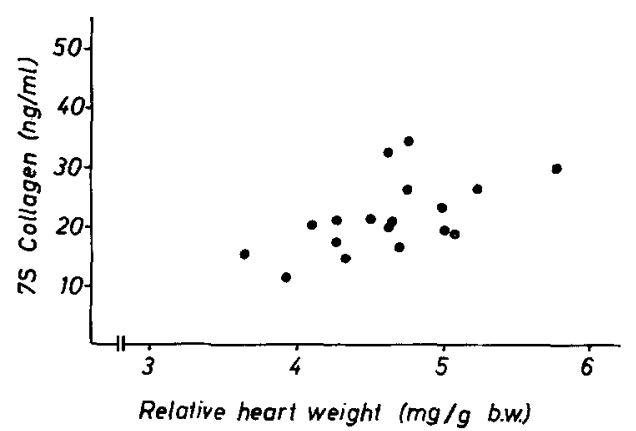

Fig. 2. Correlation between serum concentration of $7 \mathrm{~S}$ collagen and relative heart weight as an integral parameter of hypertension in nondiabetic hypertensive rats. Each point represents the value of one rat $(r=0.63 ; p<0.01)$

spectively. Diabetes was ascertained by blood glucose determinations using a glucose oxidase kit (Boehringer Mannheim, FRG) 2 and 4 weeks after streptozotocin injection. Only rats with blood glucose levels greater than $11.1 \mathrm{mmol} / \mathrm{l}$ was used. There was only a small increase in mean blood glucose levels between the second and fourth week after induction of diabetes $(16.6 \pm 0.4$ and $18.1 \pm$ $0.7 \mathrm{mmol} / 1$, respectively). Correlations were calculated using the average of these blood glucose measurements. Diabetic rats were not treated with insulin at any time. The study ended 4 weeks after diabetes induction and 8 weeks after clipping of the renal artery, respectively. At this time, animals were killed by aortic puncture. Blood was collected from each rat. After clotting at room temperature, sera were stored at $-25^{\circ} \mathrm{C}$.

Heart and kidney weight were determined with a precision balance. The increase of heart weights in the hypertensive groups was considered to be a measure of the degree myocardial hypertrophy, and thus of the chronic pressure overload. Since absolute body weights were different in diabetic and hypertensive rats, relative heart weights were used in comparison of all groups and for calculation of correlation.

\section{Determination of $7 S$ collagen and serum creatinine}

Type IV collagen concentration in the serum was determined by a radioimmunoassay specific for $7 \mathrm{~S}$ collagen $[10,11]$. This assay shows complete cross-reaction with intact type IV collagen and with the precursor form type IV collagen [15]. Intra-assay and inter-assay coefficients of variation were $2.5 \%(n=5)$ and $6 \%(n=7)$, respectively. The serum concentration of creatinine was determined using a kinetic Jaffe method (Boehringer Mannheim, FRG), which eliminates the effects of pseudocreatinines.

\section{Statistical analysis}

Differences between groups were tested with Student's t-test (twotailed probability). Linear regression analysis was chosen to calculate correlations.

\section{Results}

As shown in Table 1, normotensive diabetic rats were markedly hyperglycaemic and had lost considerable weight after streptozotocin injection (mean $72 \pm 5 \mathrm{~g}$ ). Mean absolute kidney weight was not different compared to nondiabetic controls. These findings indicate severe diabetes $[16,17]$. Similar results have been obtained in hypertensive diabetic rats. However, mean absolute weight of the unclipped kidney was significantly higher in this group than in normotensive dia- 

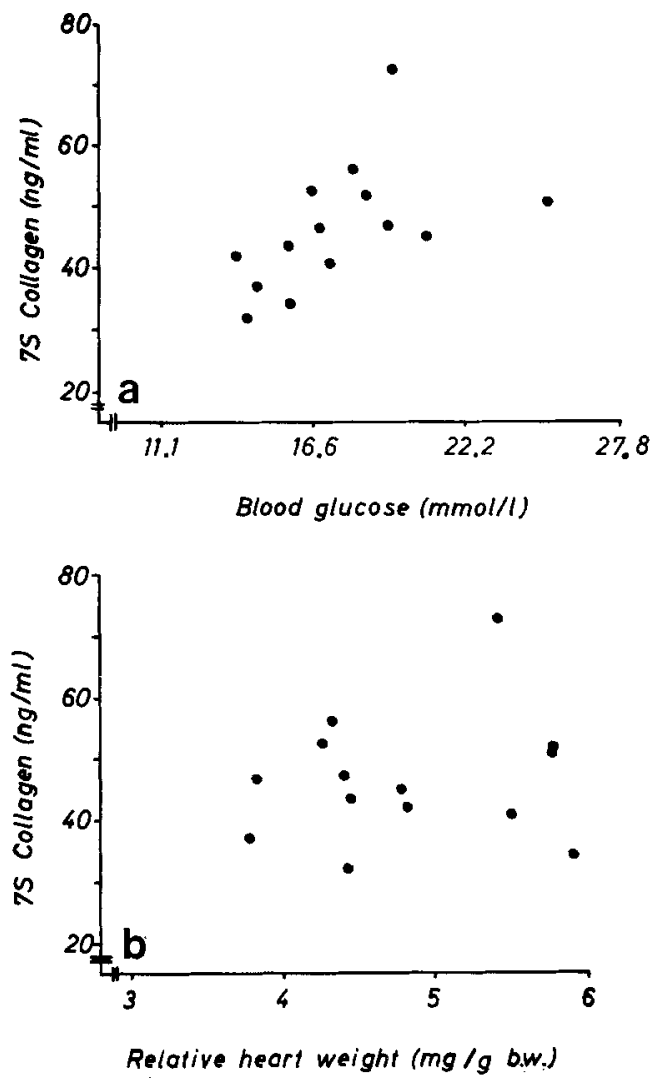

Fig.3. Correlation between (a) serum concentration of $7 \mathrm{~S}$ collagen and blood glucose and (b) relative heart weight as an integral parameter of hypertension in diabetic hypertensive rats. Each point represents the value of one rat. a: $r=0.51, p<0.05 ; \mathbf{b}: r=0.32$, NS

betic rats (Table $1, p<0.05$ ). Mean systolic blood pressure as well as absolute and relative heart weight were increased to a significant extent in diabetic and nondiabetic rats after clipping of the left renal artery (Table $1, p<0.001$ ). This increase of heart weight is attributable to "true" hypertrophy, since histological examination of the myocardial tissue disclosed the absence of fibrosis in any group [18]. No difference was noted between blood pressure and relative heart weight in hypertensive diabetic and hypertensive nondiabetic rats. Blood glucose levels and body weight were equal in diabetic rats with and without hypertension. Serum concentration of creatinine showed no difference between animals with or without clipped kidney (Table 1).

The serum concentration of $7 \mathrm{~S}$ collagen of the four experimental groups are shown in Figure 1. The mean $7 \mathrm{~S}$ collagen level of hypertensive non-diabetic rats was $51 \%$ higher than in the normotensive controls $(p<$ 0.001 ). As shown in Figure 2, a positive correlation between serum $7 \mathrm{~S}$ collagen levels and the relative heart weight could further be noted in these hypertensive non-diabetic rats $(r=0.63 ; p<0.01)$.

$7 \mathrm{~S}$ collagen concentration of the normotensive and hypertensive diabetic rats was 2.5 -fold higher than in corresponding non-diabetic animals $(p<0.01$; Fig. 1) and showed a positive correlation with blood glucose levels $(r=0.53 ; p<0.05)$, confirming earlier results [12]. When compared to normotensive diabetic animals, $7 \mathrm{~S}$ collagen levels were increased by $27 \%$ in hypertensive diabetic rats $(p<0.01$, Fig. 1$)$. A positive correlation between 7S collagen concentration and blood glucose levels was also established in hypertensive diabetic rats $(r=0.51 ; p<0.05$; Fig. $3 \mathrm{~A})$. However, no correlation could be observed between $7 \mathrm{~S}$ collagen concentrations and blood pressure or relative heart weight in this experimental group (Fig. 3 B).

\section{Discussion}

Hormonal or metabolic imbalances as well as increased permeation and deposition of plasma proteins into the capillary wall are discussed as factors influencing the metabolism of capillary basement membranes [19]. The present study indicates that haemodynamic changes also influence the metabolism of basement membrane collagen. Induction of Goldblatt hypertension leads to a significant increase of intracapillary pressure in metabolically healthy rats [8]. Using this model of hypertension, we found a marked increase of serum concentration of 7S collagen in hypertensive non-diabetic rats. Although $7 \mathrm{~S}$ collagen had not been determined in urine, this difference was probably not explained by a reduced renal excretion of $7 \mathrm{~S}$ collagen, since serum creatinine did not differ in rats with clipped and unclipped renal artery. The positive correlation between heart weight as an integral parameter of hypertension and $7 \mathrm{~S}$ collagen levels of hypertensive non-diabetic rats indicates that close relations exist between the altered haemodynamic situation and synthesis or turnover of type IV collagen in hypertensive rats. The thickening of capillary basement membrane under conditions of increased intracapillary pressure $[8,9]$ suggest that the elevated $7 \mathrm{~S}$ collagen levels are more indicative of an increased synthesis than an increased turnover of type IV collagen.

$7 \mathrm{~S}$ collagen concentration of normotensive diabetic rats was 2.5 -fold higher than in corresponding nondiabetic controls and showed a positive correlation with blood glucose, confirming earlier results [12]. It seems unlikely that this marked increase in $7 \mathrm{~S}$ collagen is related to a raised intracapillary pressure and hyperperfusion since this elevation is observed in non-insulin-treated, severely diabetic rats, whereas the elevation of intracapillary pressure and hyperfusion could only be detected in diabetic animals which were treated with small doses of insulin [3]. In addition, it has been shown that even small doses of insulin, which could not normalize the metabolic decompensation at all, already reduced $7 \mathrm{~S}$ collagen levels nearly to normal in diabetic rats [20]. These findings suggest that metabolic factors rather than haemodynamic factors are responsible for the increase in type IV collagen concentra- 
tions in diabetic rats. This increase indicates increased biosynthesis of basement membrane collagen as shown by recent experiments on basement membrane collagen synthesis, and by studies on the size distribution of type IV collagen antigens in serum [11, 12]. The normalization of elevated serum levels of $7 \mathrm{~S}$ collagen by insulin further documents that it is not a toxic non-specific side effect of streptozotocin which causes an increase in type IV collagen antigens [20].

However, hypertensive diabetic rats exhibited $7 \mathrm{~S}$ collagen serum levels which were significantly higher $(+27 \%)$ than those of normotensive rats with no difference in blood glucose and serum creatinine. It seems reasonable to assume that this indicates a further increase in type IV collagen synthesis. Histological investigations have documented a stronger increase in basement membrane-like material in kidneys of hypertensive than normotensive diabetic rats [7]. The further increase in $7 \mathrm{~S}$ collagen levels is possibly due to hyperperfusion and increase of intracapillary pressure, which has recently also been shown to occur after development of hypertension in diabetic rats [21].

The increase of $7 \mathrm{~S}$ collagen level due to hypertension in non-diabetic and diabetic rats was much less marked than the rise of $7 \mathrm{~S}$ collagen after induction of diabetes itself. Furthermore, results with hypertensive diabetic rats do not reveal a correlation with blood pressure or heart weight, but show a significant correlation with blood glucose. This again may indicate that basement membrane collagen metabolism in diabetes is influenced to a greater extent by metabolic than by additional haemodynamic factors. Nevertheless, the stimulating action of hypertension on basement membrane collagen synthesis could further explain the deleterious effect of hypertension on the course of diabetic microangiopathy, especially nephropathy. The reasons for the stimulating action of hypertension on type IV collagen synthesis in hypertensive nondiabetic and diabetic rats are still unknown. Apart from higher distension of the vessel wall or an increase of the capillary permeability $[22,23]$, an activation of the reninangiotensin system which is usually found in Goldblatt-hypertensive rats, but which is also described in diabetic patients [24], have to be discussed.

\section{References}

1. Mogensen CE (1982) Long-term antihypertensive treatment inhibiting progression of diabetic nephropathy. Br Med J 285: 685-688

2. Hasslacher Ch, Stech W, Wahl P, Ritz E (1985) Blood pressure and metabolic control as risk factors for nephropathy in Type I (insulin-dependent) diabetes. Diabetologia 28: 6-11

3. Hostetter THH, Troy JL, Brenner BM (1982) Glomerular hemodynamics in experimental diabetes mellitus. Kidney Int 19: $410-415$

4. Christiansen JS, Gammelgaard I, Frandsen M, Parving $\mathrm{HH}$ (1981) Increased kidney size, glomerular filtration rate and renal plasma flow in short-term insulin-dependent diabetics. Diabetologia 20: 451-456

5. Kohner EM, Hamilton AM, Saunders SJ, Sutcliff BA, Bulpitt CJ (1975) The retinal blood flow in diabetes. Diabetologia 11:27-33

6. Chavers B, Etzwiler D, Michael AF (1981) Albumin deposition in dermal capillary basement membrane in insulin-dependent diabetes mellitus: a preliminary report. Diabetes 30:275-278

7. Mauer SM, Steffes MW, Azar S, Sandberg SK, Brown SM (1978) The effect of Goldblatt hypertension on development of the glomerular lesions of diabetes mellitus in the rat. Diabetes 27: 738-744

8. Azar S, Johnson MA, Hertel B, Tobian L (1977) Single nephron pressures, flow and resistances in hypertensive kidneys with nephrosclerosis. Kidney Int 12: $28-40$

9. Williamson JR, Vogler NJ, Kilo C (1971) Regional variations in the width of the basement membrane of muscle capillaries in man and giraffe. Am J Pathol 63: 359-367

10. Risteli J, Rohde H, Timpl R (1981) Sensitive radioimmunoassay for $7 \mathrm{~S}$ collagen and laminin. Application to serum and tissue studies of basement membranes. Anal Biochem 113: 372-378

11. Brocks DG, Neubauer HP, Strecker H (1985) Type IV collagen antigens in serum of diabetic rats: a marker for basement membrane collagen biosynthesis. Diabetologia 28:928-932

12. Hasslacher $\mathrm{CH}$, Reichenbacher R, Gechter F, Timpl R (1984) Glomerular basement membrane synthesis and serum concentration of type IV collagen in streptozotocin-diabetic rats. Diabetologia 26: $150-154$

13. Möhring J, Petri M, Szokol M, Haack D, Möhring B (1976) Effects of saline drinking on malignant course of renal hypertension in rats. Am J Physiol 230: 849-857

14. Byron FB, Wilson C (1938) A plethysmographic method for measuring systolic blood pressure in the intact rat. J Physiol 301-304

15. Timpl R, Wiedemann $H$, van Delden V, Furthmayr H, Kühn K (1981) A network model for the organization of type IV collagen molecules in basement membranes. Eur J Biochem 120:203-211

16. Seyer-Hansen K (1978) Renal hypertrophy in experimental diabetes: a comparison to compensatory hypertrophy. Diabetologia 14: $325-328$

17. Brekke IB, Gundersen HJG, Østerby R (1985) Thickening of glomerular basement membrane in rats with severe diabetes without kidney hypertrophy. Diab Nephropathy 4: 19-22

18. Mall G, Mattfeld T, Hasslacher Ch, Mann J (1986) Morphological reaction patterns in experimental cardiac hypertrophy - a quantitative stereological study. Basic Res Cardiol [Suppl] 81: 193-201

19. Williamson JR, Kilo C (1977) Current status of capillary basement membrane disease in diabetes mellitus. Diabetes 26:65-73

20. Risteli J, Draeger KE, Regitz G, Neubauer HP (1982) Increase in circulating basement membrane antigens in diabetic rats and effects of insulin treatment. Diabetologia 23: 266-269

21. Bank N, Klose R (1985) Glomerular pressure in rats with diabẹtes and hypertension. Kidney Int 28: 241 (Abstract)

22. O'Hare JAO, Ferriss JB, Twomey B, O'Sullivan DJ (1983) Poor metabolic control, hypertension and microangiopathy independently increase the transcapillary escape rate of albumin in diabetes. Diabetologia 25: 260-263

23. Parving HH, Gyntelberg F (1973) Transcapillary escape rate of albumin and plasma volume in essential hypertension. Circ Res 32: $643-651$

24. Drury PL, Bodansky HJ, Oddie CJ, Cudworth AG, Edwards CRW (1982) Inereased plasma renin activity in type I diabetes with microvascular disease. Clin Endocrinol 16: 453-461

Received: 17 September 1986

and in revised form: 3 March 1987

Professor C. Hasslacher

Medical University Clinic

Bergheimer Straße 58

D-6900 Heidelberg

FRG 